\title{
Clinical Correlates and Electroencephalographic Characteristics of Two Additional Patterns Related to 14 and 6 per Second Positive Spikes
}

\author{
J. Reiher and L. Carmant
}

\begin{abstract}
Two additional patterns, minuscule 28 per second positive spikes and huge $\mathrm{N}$-shape potentials, have been identified exclusively in the EEGs of patients with 14 and 6 per second positive spikes. They occur predominantly during drowsiness and light sleep, usually in children, seldom in young adults. Their presence adds little to the clinical relevance of positive spikes. Familiarization with the $\mathrm{N}$-shape potentials - the commoner of the two patterns - is important, lest they are mistaken for interictal abnormalities of significance such as atypical spike-wave complexes.
\end{abstract}

RÉSUMÉ: Corrélations cliniques et caractéristiques électroencéphalographiques de deux grapho-éléments reliés aux pointes positives à 14 et $6 \mathrm{~Hz}$ Chez 100 sujets de pointes positives à 14 et $6 \mathrm{~Hz}$, une revue des tracés EEGs a permis d'indentifier deux grapho-éléments supplémentaires. Des pointes positives minuscules à $28 \mathrm{~Hz}, \mathrm{~d}$ 'une part, et des potentiels en forme de $\mathbf{N}$, considérablement plus amples, d'autre part, se retrouvent en effet, principalement chez l'enfant, durant la somnolence et le sommeil léger. Nonobstant leur signification plus que restreinte, il importe néanmoins de ne pas méprendre le potentiel $\mathbf{N}$ pour un complexe pointe-onde atypique.

Can. J. Neurol. Sci. 1991; 18: 488-491

Over the years, two additional patterns, 28 per second positive spikes and $\mathrm{N}$-shape potentials, have been observed in our laboratory in the EEGs of patients with 14 and 6 per second positive spikes. The present study was undertaken to outline their electroencephalographic characteristics and clinical significance, and to verify whether they bear an exclusive relationship to positive spikes.

\section{Material ANd Methods}

The awake and sleep EEGs of 100 patients with 14 and 6 per second positive spikes and of 100 matched controls obtained between 1972 and 1989 were randomly selected and reviewed. They were screened for the presence of both study patterns. The following parameters were analyzed: amplitude, morphology, duration, frequency of occurrence, polarity, spatial and temporal distribution, and relation to 14 and 6 per second positive spikes. Age, sex and chief referral complaints were also recorded.

Availability of 16- or 18-channel recordings during both wakefulness and sleep using scalp to scalp and ear referential montages was mandatory in all patients and controls for inclusion in the study.

\section{RESULTS}

There were 63 males and 37 females. The mean age of patients (in years) with isolated 6 per second positive spikes was higher (24) than with either isolated 14 per second positive spikes (11) or combined 14 and 6 per second positive spikes (14).

In 6 patients, and in none of the controls, minuscule repetitive 28 per second positive spikes occur in bursts of less than $400 \mathrm{msec}$ (Figure 1). They are always intermingled with 14 per second positive spikes, smaller in amplitude but similar in distribution. Nonrepetitive single fast transients occurring in association with a burst of 14 or 6 per second positive spikes were discounted, even though other parameters including amplitude, morphology, polarity and distribution otherwise conformed to those of repetitive 28 per second positive spikes.

In 28 patients, and in none of the controls, single, widely distributed and stereotyped diphasic $\mathrm{N}$-shape potentials were observed (Figures 2, 3 and 4). Measuring up to $200 \mu \mathrm{V}$, they usually follow a burst of 14 to 6 per second positive spikes and then a single negative spike. The initial component lasts 200

\footnotetext{
From the Department of Neurology, University of Sherbrooke, School of Medicine, Sherbrooke, Quebec

Received January 22, 1991. Accepted in final form May 27, 1991

Presented at the annual meeting of the American EEG Society, September 23, 1990, Houston, Texas

Reprint requests to: Jean Reiher, M.D., Neurology Department, Centre Hospitalier Universitaire of Sherbrooke, 3001 - 12th Avenue N., Sherbrooke, Quebec, Canada J1H 5N4
} 
msec. Its polarity is opposite that of the preceding positive spikes and that of the late component. The latter extends over one second, often overridden by low amplitude theta waves. Display of the entire N-shape potential is optimal in those com- parison derivations linking homologous electrodes across the midline (Figures 2 and 4). More than one phase reversal is commonly observed in scalp to scalp linear montages (Figure 3). In referential ear derivations, polarity of the $\mathrm{N}$-shape potential is

$$
\text { DAs }
$$

Figure 1-28 per second positive spikes intermingled with 14 per second positive spikes.

01-02

T5-T6

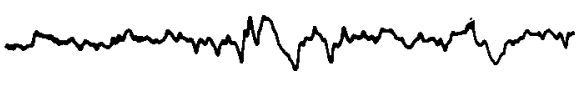

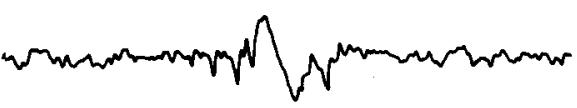

A1-A2

T3-T4

F7-F8

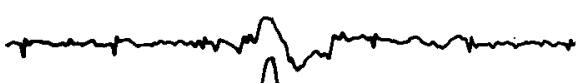

FP1-FP2
FP2-FP1

F8-F7

T4-T3

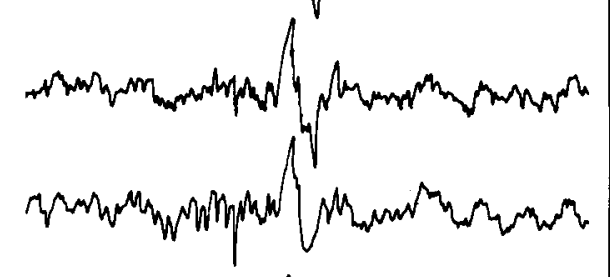

A2-A1

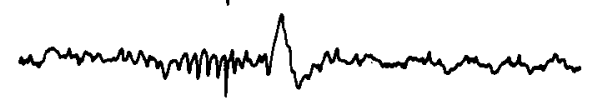

T6-T5

$02-01$

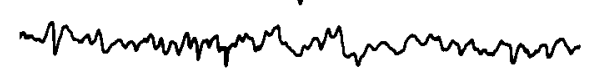

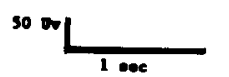

Figure $2-N$-shape potentials, preceded by 6 per second positive spikes (on the left) and by 14 per second positive spikes (on the right) in comparison derivations linking homologous electrodes across the midline. 
ubiquitously uniform on the scalp (Figure 3). Its amplitude fluctuates more than once in successive exploring electrodes from frontopolar to occipital regions through temporal and parasagittal areas. The N-shape potential occurred 51 times out of 55 in association with a burst of either $14 / \mathrm{sec}$ ( 44 times), $6 / \mathrm{sec}$ ( 6 times) or $28 / \mathrm{sec}$ (once). Average number of $\mathrm{N}$-shape potentials amounts to 1.96 per recording.

Minuscule 28 per second positive spikes and larger N-shape potentials are found predominantly in children and teenagers. They occur unilaterally or bilaterally, synchronously or asynchronously, much more often during light sleep than during wakefulness, and least during deeper stages of sleep. Exceptionally, in 4 patients, both patterns coexist in the same recording.

Seizure, headache, behavioral disorder, syncope, infection and head injury were the most common reasons for referral in patients of the study group as in controls (87 times out of 100 in both groups). A similar prevalence was observed in the subgroup of patients with the 28 per second positive spikes and the $\mathrm{N}$-shape potentials.

Phantom 6/sec. spike-wave complexes were identified in 2 controls and in 8 patients.

\section{Discussion}

The morphologically distinct 28 per second positive spikes and the $\mathrm{N}$-shape potentials have thus been identified exclusively in the EEGs of patients with 14 and 6 per second positive spikes, and not at all in those of the control group.

The 28 per second positive spikes can readily be overlooked because of their very low amplitude. Even Hughes ${ }^{1}$ who has scrutinized the harmonic relations between the 14 and 6 per second, or 14 and 7 per second positive spikes, as he calls them, makes no mention of additional harmonics nor of subharmonics.

Reason for ignoring the huge $\mathrm{N}$-shape pattern is more obscure. An incidental illustration of the phenomenon in a referential ear montage may already be recognized in the 1952 Gibbs' Atlas ${ }^{2}$ in association with a burst of 6 per second positive spikes. Referential ear montages are however not mandatory for their display. Longitudinal bipolar montages and montages including both scalp to scalp and referential ear derivations are indeed equally appropriate to illustrate both 14 and $6 \mathrm{~s}$ and $\mathrm{N}$-shape potentials. The latter montages underscore their wide distribution over the scalp. They also better display the components that must be properly identified if the $\mathrm{N}$-shape potentials are not to be confused with atypical spike-wave complexes (Figure 4).

Silverman ${ }^{3}$ has reported an exceedingly common association between 14 and 6 per second positive spikes and so-called typical and atypical phantom $6 / \mathrm{sec}$. spikes-wave complexes. His illustration of the atypical form may very well represent a single 28 per second positive spike followed by a 6 per second positive spike. Our observations of a single negative spike intercalated between a burst of positive spikes and an $\mathrm{N}$-shape potential

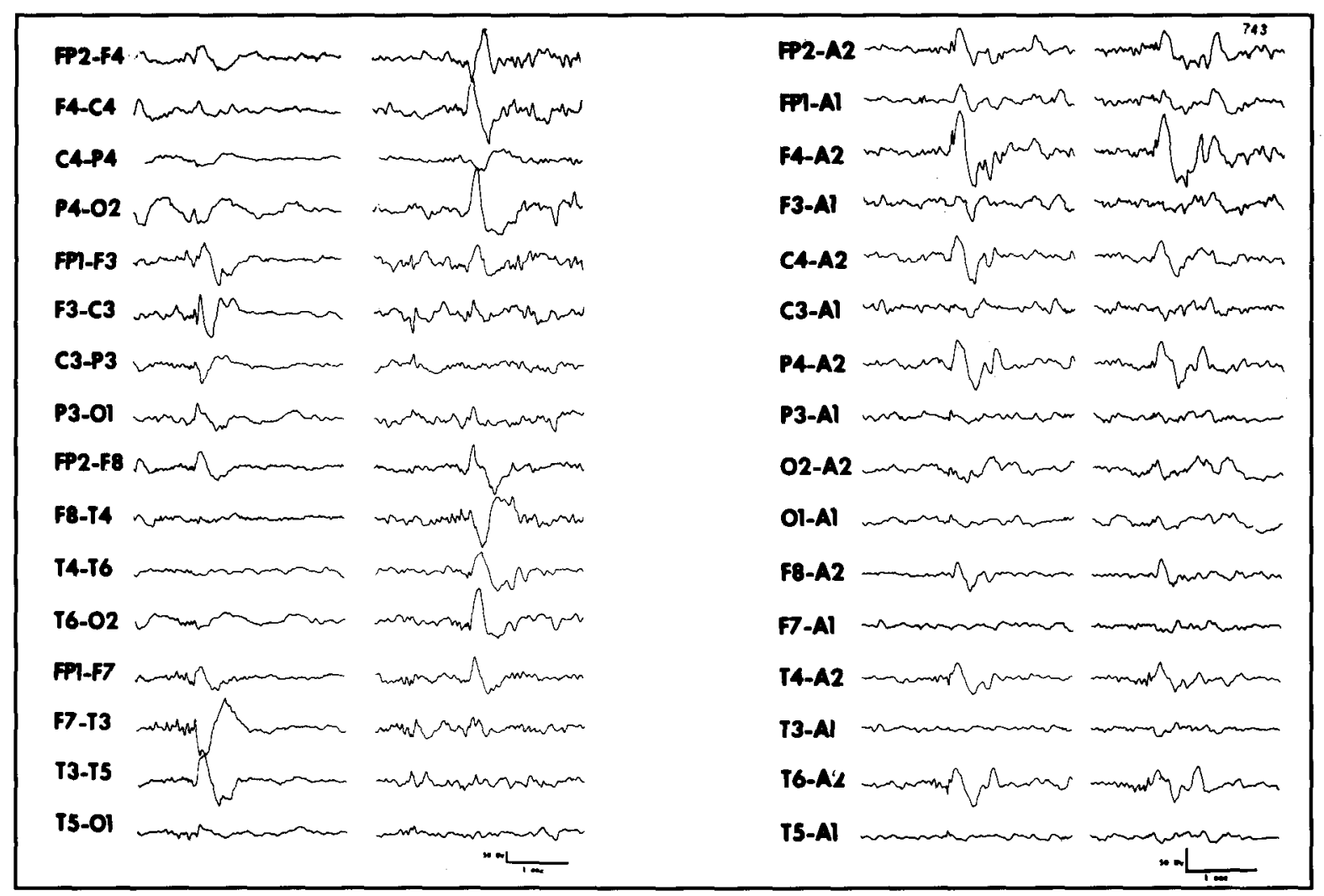

Figure 3-N-shape potentials exhibiting multiple phase reversals in scalp to scalp linear montages, an oscillating amplitude from frontopolar to occipital regions and a uniform polarity in referential ear derivations. 


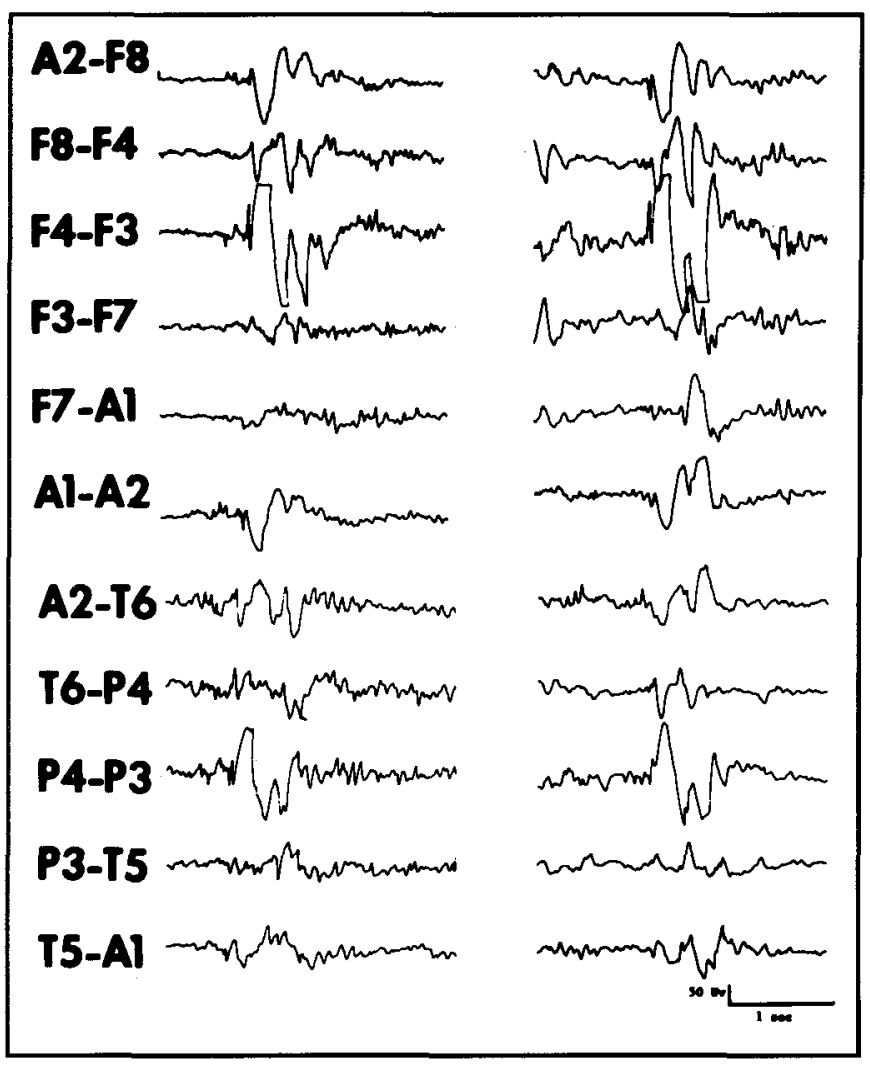

Figure $4-H u g h ~ N$-shape potentials and overriding theta waves are best seen in $F_{4}-F_{3}$ and $P_{4}-P_{3}$ derivations. They follow a burst of 14 per second positive spikes. They are immediately preceded by a single negative spike. They should not be mistaken, however, for an atypical spikewave complex.
(Figure 4) needs to be distinguished from a typical or atypical phantom spike-wave complex. Indeed the juxtaposition of a high amplitude spike to the $1000 \mathrm{msec} \mathrm{N}$-shape component on one hand, unilaterally or asynchrony of the entire complex on the other hand, constitute the cardinal attributes of a pattern that differs considerably from either Silverman's typical $200 \mathrm{msec}$ phantom spike-wave complex or from Hughes' larger "WHAM" variety of 6 per second spike-wave pattern. ${ }^{4}$

No significant difference in chief referral complaints has been observed between patients and controls. This finding is not unexpected, since positive spikes have been shown to represent normal variants. 5,6 The lack of a distinctive clinical profile in the subgroup of patients with 28 per second positive spikes and $\mathrm{N}$-shape potentials is no more surprising since both patterns do bear an exclusive relationship to 14 and $6 / \mathrm{sec}$ positive spikes.

Lack of clinical significance notwithstanding, familiarization with the N-shape pattern, the commoner of the two, is important to avoid misconstructions not only with normal sleep potentials such as K-complexes, but more importantly with atypical spikewave complexes (Figure 4).

\section{REFERENCES}

1. Hughes JR. The 14 and 7 per second positive spikes - A reappraisal following a frequency count. Electroencephalogr Clin Neurophysiol 1960; 12: 495-496.

2. Gibbs FA, Gibbs EL. Atlas of electroencephalography, 1952: 2, Addison-Wesley, Cambridge, MA.

3. Silverman D. Phantom spike-waves and the fourteen and six per second positive spike pattern: a consideration of their relationship. Electroencephalogr Clin Neurophysiol 1967; 23: 207-213.

4. Hughes JR. Two forms of the 6 per second spike and wave complex. Electroencephalogr Clin Neurophysiol 1980; 48: 535-550.

5. Lombroso CT, Schwartz IH, Clark EM, et al. Ctenoids in healthy youths: controlled study of 14- and 6-per second positive spiking. Neurology 1966; 16: 1152-1158.

6. Long MT, Johnson LC. Fourteen- and six-per-second positive spikes in a non-clinical male population. Neurology 1968; 18: 714-716. 